\title{
Functional connectivity hubs and thalamic hemodynamics in Rolandic Epilepsy
}

\author{
Caroline Garcia Forlim ${ }^{1,2 *}$, Roma Siugzdaite ${ }^{1}$, Yang Yu ${ }^{3}$, Ye-Lei Tang ${ }^{4}$, Wei Liao ${ }^{5,6,7}$, Daniele \\ Marinazzo $^{1^{*}}$
}

1. Department of Data Analysis, Faculty of Psychology and Pedagogical Sciences, University of Ghent, B-9000 Ghent, Belgium

2. Clinic and Policlinic for Psychiatry and Psychotherapy, University Medical Center Hamburg-Eppendorf, Hamburg, Germany

3. Mental Health Education and Counseling Center, Zhejiang University, Zhejiang, China

4. Department of Neurology, the Second Affiliated Hospital of Medial College, Zhejiang

University, Zhejiang, China

5. Center for Information in BioMedicine, Key Laboratory for Neuroinformation of Ministry of Education, School of Life Science and Technology, University of Electronic Science and Technology of China, Chengdu 610054, China

6. Center for Cognition and Brain Disorders and the Affiliated Hospital, Hangzhou Normal University, Hangzhou 310015, China

7. Zhejiang Key Laboratory for Research in Assessment of Cognitive Impairments, Hangzhou310015, China

*Correspondence to: c.garcia-forlim@uke.de (CGF), daniele.marinazzo@ugent.be (DM)

\begin{abstract}
Our brain is a complex organ with different levels of interaction and therefore can be thought as a complex network. In high level interactions, the brain network is formed by interconnected areas called nodes and their connections are the links. Hubs play a key role in information processing in the brain. Brain networks can be extracted using several imaging modalities, here we focus on networks based on resting state fMRI (rsfMRI) where spontaneous brain activity is indirectly measured resulting in a blood-oxygenlevel dependent (BOLD) signal for each voxel. Brain regions or voxels are said to be functionally connected and therefore, a link exists, if they present temporal correlation. The advantages of rsfMRI are the easiness of the acquisition suitable for children and clinical population and to be able to uncover networks related to spontaneous or "default mode" of the brain. Moreover, it has been shown that the resting state networks are impaired in psychiatric and neurological disorders.
\end{abstract}

Rolandic epilepsy (RE) is one of the most common epilepsy in childhood manifesting abnormal EEG activity in central-temporal areas. Despite seizure remission during adolescence, recent studies have shown a serious of comorbidities. Moreover, the risk of cognitive impairments has been linked to interictal epileptic discharges (IED). Nevertheless, the underlying mechanisms are not fully understood.

Here, we applied two novel methods to resting state fMRI, the blind deconvolution method to recover the neural activity and to extract the hemodynamic response (HRF) and functional connectivity density (FCD). FCD is a data-driven voxel-wise new tool combining graph theory and functional connectivity that 
unveils densely connected regions that can work as functional hubs of information in the brain. The goal was to identify hubs of information flow and possible network disruption in RE in patients with and without IEDs.

FCD maps revealed main hubs in the posterior cingulate, precuneus, cuneus and calcarine. Patients with IEDs during the scanner showed higher FCD as compared to healthy controls and larger hub in the postcentral precentral gyri, key focal areas in RE. Patients with no IEDs during the scanner showed overall lower FCD as compared to controls and IED groups. Group comparison revealed hyper local connectivity in bilateral thalamus in the patients with IEDs compared to patients without IEDs. Additional exploratory HRF analysis showed that patients with IEDs presented higher response height in the HRF in the thalamus evidencing the inhomogeneity of the HRF among groups.

We speculate that locally abnormal information flow in bilateral thalamus might suggest the involvement of this region in the generation of spikes in RE. It also provides additional evidence for an epileptic as a network disease rather than a focus dysfunction. This hypothesis could be further confirmed in meta analysis, small group size is the main limitation of this study. To the best of our knowledge, this is the first study to combine blind deconvolution and FCD to the whole brain analysis in RE.

\section{Introduction}

Our brain is a complex organ with different levels of interaction and connections and therefore can be thought as a complex network. In high level interactions, the brain network is formed by interconnected areas called nodes and their connections are the links. Due to the nature of the interactions in the brain, the optimal network should be able to process locally specialized information as well as to connect localized information centres among themselves. In this way, information can travel globally and fast in the brain. Hubs play a key role in this interplay.

The brain networks can be calculated using several imaging modalities as functional magnetic resonance imaging (fMRI), structural magnetic resonance imaging, electroencephalogram (EEG), diffusion tensor imaging (DTI) among others. Here we focus on networks based on resting state fMRI (rsfMRI) where spontaneous brain activity is indirectly measured resulting in a blood-oxygen-level dependent (BOLD) signal for each voxel. Voxels $\left(\sim \mathrm{mm}^{3}\right)$ are the minimum brain unity that can be measured by the fMRI. In rsfMRI, the subjects lie in the scanner from 5 to $10 \mathrm{~min}$ and are instructed to relax. Brain regions or voxels are said to be functionally connected and therefore, a link exists, if they present temporal correlation. Typically, links between areas or voxels are inferred using Pearson's correlation coefficient. The main advantages of rsfMRI are the easiness of the acquisition suitable for children and clinical population and to be able to uncover networks related to spontaneous or "default mode" of the brain. Moreover, it has been shown that the resting state networks are impaired in psychiatric and neurological disorders. 
Epilepsy is one the most common neurological disorders and it is characterized by unpredictable seizures, atypical oscillatory dynamics and sychronization across brain regions [1]. Rolandic epilepsy (RE) is idiopathic childhood epilepsy, with a typical onset at age of $7-10$ years. Seizures can be infrequent and usually occur at night during sleep. Despite seizure remission during adolescence, recent studies have shown a series of comorbidities in attention [2], language [3], motor performance [4], memory [5]. The risk

of cognitive impairments has been linked to interictal epileptic discharges (IED)[2,6,7] that are abnormal waveforms not associated with seizure symptoms. Nevertheless, the underlying mechanisms are not fully understood [6].

The most used tools to investigate epilepsy are EEG and fMRI. EEG is widely used to characterize the abnormal electrical activity present in the brain signal of epileptic patients and to identify its source, while fMRI, due to its high spatial resolution is helping to uncover subcortical structures involved in epilepsy as well as whole brain connectivity. Resting state fMRI is a relatively young technique that is particularly suitable for the study of epilepsy, especially when working with children, due to the ease of data acquisition.

In resting state fMRI, as opposed to the task fMRI, the spontaneous events of the brain activity are measured. Although the invaluable contributions of the fMRI, little concern is taken about the nature of the signal in this technique. What is in fact being measured is an indirect secondary effect of the real neural activity in which there is a hemodynamic delay due to the fact that it takes several seconds to the changes in the oxygen level in the tissues to be perceived in the MRI [8,9]. It results in a measured signal that is delayed, relative to the real neural activity, causing a temporal blurriness. In addition, the hemodynamic response (HRF) is most of the times considered to have a canonical shape that is homogeneous across brain regions. Nevertheless, there is evidence that this is not the case for epilepsy [10-14]. All of these technical inaccuracies together can lead to distorted and blurred time series. The common way to account for those is performing a deconvolution.

Deconvolution has proven to be a good technique to overcome these problems applied for event- related fMRI diminishing the inherent temporal blurring in fMRI, hence increasing the temporal precision of the neural activity time course [8]. The same method was adopted for resting state data and called blind deconvolution $[15,16]$. In the blind deconvolution events are blindly obtained from BOLD signals. Once the signal is deconvolved, the following step is the choice of the most suitable analysis methods. Often in resting state fMRI, functional connectivity (FC) is chosen.

Classical FC assesses only global correlations and it faces issues as the choice of a seed. Choosing the seed, a strong a priori hypothesis is needed and may bias the outcome. To overcome these issues, we used a new technique named functional connectivity density (FCD) which combines functional connectivity and graph theory. FCD investigates voxel-wise local and large-scale network unveiling important global and local hubs of information by clustering functional correlations in the whole brain $[17,18]$. 
Here, firstly we applied a blind deconvolution method for resting state fMRI to estimate the neural signal with increased the temporal precision and to extract the HRF for posterior exploratory analysis. Secondly, we applied FCD to the neural signal to identify particularities of local and global networks involved in Rolandic epilepsy.

\section{Methods}

\section{Participants}

Children with RE were recruited from the Second Affiliated Hospital of Zhejiang University School of Medicine, China. Patients were diagnosed by experts based on ILAE classification [19], the presence of simple partial tonic-clonic seizures during sleep, and spike-waves in centrotemporal regions. Exclusion criteria were focal brain lesions, falling asleep during scanning, or head motion exceeding $3 \mathrm{~mm}$ in translation or 3 degrees in rotation. The whole resting-state fMRI run was used for each subject. Healthy controls were recruited from local primary schools in Hangzhou, China. These subjects had no history of neurologic disorders, psychiatric illnesses or gross abnormalities on brain MR images.

After the exclusion criteria this study involved 27 children with Rolandic epilepsy, 15 of which presented interictal epileptiform discharges (IEDs), 12 non-IED and 11 age-matched healthy controls (HCs) (Table1). The division of IED and nonIED group were performed based on spikes present in the EEG recordings. For all subjects in the non-IED group, there were no spikes during the total length of the EEG-fMRI simultaneous recordings. All subjects in the IED group presented spikes during the EEG-fMRI recordings.

Table 1. Clinical data

\begin{tabular}{lllll}
\hline & IED & non-IED & HC & P value \\
\hline Age (years) & $8.93 \pm 1.71$ & $10.25 \pm 2.26$ & $8.73 \pm 1.95$ & $0.1344^{\mathrm{a}}$ \\
Sex (females:males) & $10: 5$ & $5: 7$ & $6: 5$ & $0.4395^{\mathrm{b}}$ \\
Onset age (years) & $7.80 \pm 1.21$ & $7.42 \pm 2.71$ & & $0.6273^{\mathrm{c}}$ \\
Side of EEG (R:L:B) & $9: 5: 1$ & $6: 4: 2$ & $0.6977^{\mathrm{e}}$ \\
Number of seizures (/year) & $2.25 \pm 1.36$ & $9.64 \pm 15.53$ & & $0.2171^{\mathrm{d}}$ \\
Treatment:Naïve & $5: 10$ & $10: 2$ & $0.0094^{\mathrm{e}}$ \\
Medication (LEV:VAL:LTG:OXC) & $0: 2: 3: 1$ & $5: 3: 4: 1$ & & $0.3621^{\mathrm{e}}$ \\
Mean FD & $0.19 \pm 0.04$ & $0.16 \pm 0.02$ & $0.13 \pm 0.01$ & $0.2659^{\mathrm{a}}$ \\
\hline${ }^{a}$ One-way ANOVA. & & & \\
b Kruskal-Wallis ANOVA. & & & \\
c two-sample T test. & & & & \\
d Mann Whitney U-test. & & & \\
e Chi-square test. & & & &
\end{tabular}

\section{MRI data acquisition}

Brain imaging data were acquired on 3T MRI scanner (GE discovery $750 \mathrm{MRI}$ ) and using a standard head coil at the Center of Cognition and Brain Disorders, Hangzhou Normal University, China. Foam pads were used to reduce head motion and scanner noise. All participants were asked to hold still, with eyes closed, not to think of anything in particular. The rsfMRIs were acquired using an EPI sequence, TR/TE $2000 \mathrm{~ms} / 30 \mathrm{~ms}$, and flip angle $90^{\circ}$ and EPI sequence with 26 axial slices of $3.2 \mathrm{~mm}$ thickness, with in-plane 
resolution $64 \times 64$ and TR/TE $=2000 \mathrm{~ms} / 30 \mathrm{~ms}$, FOV 220x 220mm, no interslice gap, 240 volumes. Subsequently, T1-weighted anatomical images were acquired in the sagittal orientation using a MPRAGE sequence $\left(\mathrm{TR} / \mathrm{TE} 8.06 \mathrm{~ms} / 3.136 \mathrm{~ms}, \mathrm{FA} 8^{\circ}, \mathrm{FOV}=256 \times 256 \mathrm{~mm}^{2}\right.$, matrix $=256 \times 256$, slice thickness $=1$ $\mathrm{mm}$, no interslice gap, and 176 slices).

\section{Preprocessing}

To ensure for steady-state longitudinal magnetization, the first 10 images were excluded. The remaining 230 images were corrected for slice timing and realigned. Individual T1 was coregistered to functional images. Then anatomical images were segmented into gray matter, white matter, and cerebrospinal fluid; spatially normalized with voxel size $3 \mathrm{~mm}$ to the MNI template. The functional images were spatially smoothed with a 6-mm FWHM. All above mentioned preprocessing steps were performed using DPARSF http://rfmri.org/DPARSF, [20]. Afterwards, the signal from the whole brain, white matter and cerebrospinal fluid was regressed; temporal band-pass filtering $(0.01-0.1 \mathrm{~Hz})$ was applied using REST toolbox [21]. Finally, detrending was done with SPM12. We also calculated Framewise displacement (FD) [22] to account for movement.

\section{HRF signature extraction and blind deconvolution for resting state fMRI}

Deconvolution has proven to be a good technique for diminishing the inherent temporal blurring in fMRI, hence increasing the temporal precision of the neural activity time course [8]. The blurring is due the fact that the fMRI signal is solely a secondary measure of the real neural activity where there is a hemodynamic time lag.

The neural activity can be either task related or spontaneous [23]. In resting state studies, as opposed to the task fMRI, the neural events are not explicit. Moreover, there is an increase believe that the relatively large amplitude BOLD signals might encode important information [24].

We applied a more general deconvolution method for resting state called blind deconvolution $[15,16]$. In this method, events were obtained from peaks of the brain spontaneous activity from BOLD signal, signatures from resting state HRF were extracted from BOLD peaks, as in Wu et al. (2013, 2015). HRF was estimated for each voxel using Finite impulse response (FIR) function rather than a canonical HRF since recent work showed that canonical HRF may not be suitable for epilepsy [10]. To analyze possible inhomogeneities in the hemodynamic activity we extracted 3 features from the HRF: response height, time to peak and full width at half maximum (FWHM) as measures for magnitude, latency and duration. The extracted HRF was then used to deconvolve the BOLD time series to obtain the neural signal. The code for

the HRF blind deconvolution is freely available at http://users.ugent.be/ dmarinaz/HRF_deconvolution.html

Functional connectivity density mapping (FCD)

Functional connectivity density (FCD) is a data-driven voxel-wise new graph theory tool $[17,18]$ that unveils densely connected regions that can work as functional hubs of information in the brain network. By adding at the voxel-level graph measures as degree, strength and cluster to the classic FC, FCD takes a step further in the basic network construction. 
Firstly FCD calculates the links given by Pearson's correlation coefficient between one voxel $x_{0}$ and the other voxels $x_{1}, x_{2}, \cdots, \mathrm{x}_{n}$ in the brain and a MRI signal-to-noise threshold $\mathrm{T}$, only statistically significant correlation links with correlation greater than 0.6 and $\mathrm{T}=50$ are kept [17,18]. To compute local FCD, the number of significant functional connections per voxel in the local cluster, $\mathrm{k}$, is inferred using a threedimensional searching algorithm developed in IDL (ITT Visual Information Solutions). If a voxel $x_{1}$ was functionally connected to $x_{0}$ and adjacent to a voxel linked to $x_{0}$ by a continuous path of functionally connected voxels, $x_{1}$ was added to the local cluster of $x_{0}$. The local FCD at $x_{0}$ was the total number of elements in the cluster (Fig. 1)

Global FCD is the local degree of each voxel, disregarding cluster restrictions and is defined as $N \times(N-1) / 2[17]$.

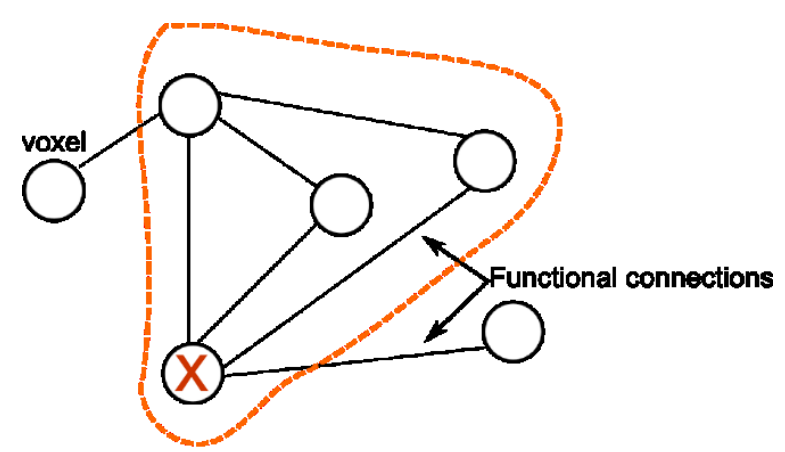

Local cluster of voxel $X$

$F C D$ in voxel $x=3$

Fig. 1. Local voxel cluster in FCD .

\section{Statistics}

The local and global FCD maps were spatially smoothed $6 \mathrm{~mm}$ FWHM in SPM 12 to minimize differences across subjects in brain anatomy according to [18]. For statistical group analysis, one-way ANOVAs were performed in SPM 12 and a threshold of $\mathrm{p}<0.05$ family-wise error (FWE) correction on a cluster level was considered. Movement given by framewise displacement [22], age and gender were included as covariates.

\section{Results}
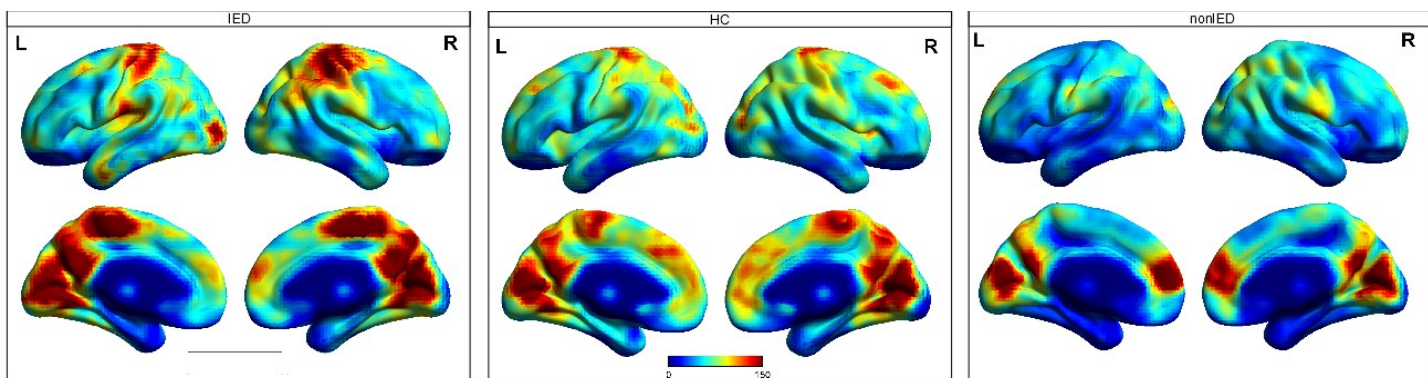
Fig. 2. Local FCD within group map showed hub regions where voxels were functionally connected to their neighbors.

Before applying FCD, the neural signal was estimated by a blind deconvolution. This procedure allows for signals with increased temporal precision and, in addition, provides the HRFs for posterior exploratory analysis. The resulting neural signal was used to calculate the FCD, a new voxel-wise data-driven method that combines the classical FC with graph analysis. We applied FCD in 3 groups: epileptic patients with IEDs during the scan, epileptic patients without IEDs during the scan and healthy controls. FCD revealed hub regions in which voxels were continuously functionally connected to their neighbors (Fig 2). The mean local FCD of the control group revealed hubs in the posterior cingulate, precuneus, cuneus, calcarine. The IED group had higher FCD than the healthy controls and larger hub in the postcentral precentral gyri. In turn, the non IED group showed lower FCD than healthy controls for most brain regions, with exception of superior medial frontal.

Our FCD group analysis revealed an increase in strength of local (or also called short-range) functional connectivity density in patients with IED in bilateral thalamus as compared to non-IED patients (Fig.3A, Table 2). Other comparisons IED versus HC and non-IED versus HC did not show any differences as well as for global FCD.

The HRF group analysis between IED and non-IED showed bigger response height to IED group in left thalamus (Fig. 3B; table 2). The response height in the average voxel-specific HRF $(-9,-21,6)$ across subjects were 3 times higher for the IED group compared to the non-IED (Fig. 1C). The HRF for the nonIED group was delayed as compared to the IED group: time to peak for the non-IED was $12 \mathrm{~s}$ and for the IED was $10 \mathrm{~s}$. The same analysis above was done using canonical HRF instead of FIR (see section $H R F$ signature extraction and blind deconvolution for resting state fMRI ) and no group differences were found.
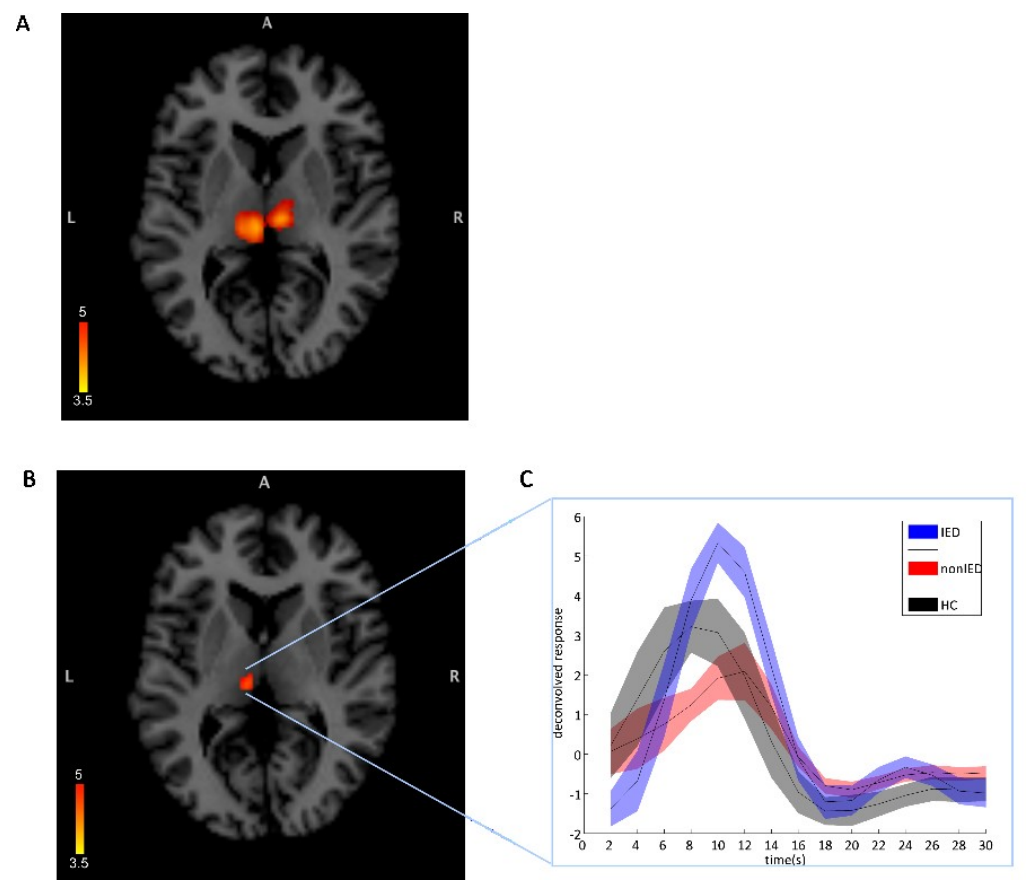

Fig. 3. A - Higher local FCD in bilateral thalamus in the IED group as compared to the non-IED group. B - Higher response height in the left thalamus for the IED group as compared to the non-IED group. C - Average Hemodynamic response function (FIR) for IED and non-ED groups 
Table 2. Clusters with significant differences between groups.

\begin{tabular}{|c|c|c|c|c|c|}
\hline REGION & $\mathrm{X}$ & $\mathrm{Y}$ & $\mathrm{Z}$ & $\mathrm{T}$ & $\begin{array}{l}\text { Voxels in } \\
\text { the cluster }\end{array}$ \\
\hline \multicolumn{6}{|l|}{$\begin{array}{l}\text { IED }>\text { nonIED } \\
\text { (cluster level FWE, } p=0.005, n=270 \text { ) }\end{array}$} \\
\hline Thalamus L & -3 & -24 & 6 & 4.53 & 89 \\
\hline Thalamus R & 9 & -21 & 0 & 5.07 & 80 \\
\hline Brainstem R & 6 & -27 & -6 & 5.09 & 59 \\
\hline \multicolumn{6}{|l|}{$\begin{array}{l}\text { HRF - Response height (ANOVA) } \\
\text { (cluster level FWE, } p=0.013, n=9 \text { ) }\end{array}$} \\
\hline Thalamus L & -9 & -21 & 6 & 4.04 & 9 \\
\hline
\end{tabular}

\section{Discussion}

The brain can be thought as a complex network. In high level interactions, the brain network is formed by interconnected areas called nodes and their connections are the links. Hubs play a key role in information processing in the brain. The brain networks can be calculated using several imaging modalities, here we focused on networks based on resting state fMRI (rsfMRI) where spontaneous brain activity is indirectly measured. The main advantages of rsfMRI are the easiness of the acquisition suitable for children and clinical population and to be able to uncover networks related to spontaneous or "default mode" of the brain. Moreover, it has been shown that the resting state networks are impaired in psychiatric and neurological disorders.

We calculated by means of the FCD the distribution of hubs in brain of the healthy controls and patients with RE. FCD is a robust and reliable measure that reveals densely connected regions in the brain that can work as hubs of information processing. The FCD for the control group revealed similar map as found in meta study by Tomasi and Volkow, [18] with high FCD in the posterior cingulate, precuneus, cuneus, calcarine. Patients who had IEDs during the scanner presented in average hyper connected map. Moreover, a large hub was seen in the postcentral/precentral gyrus. This result expected because these regions are known to be a focal area in RE. Surprisingly, patients that did not present IEDs during the scanner presented in average a hypo connected map. In the group level, we found higher local FCD in bilateral thalamus in patients with IEDs compared to non-IEDs, which means a hyper-connected local network in this region, where more information is being exchanged within thalamus and local surroundings during abnormal electrical activity in the brain. Thalamus is a key area in the brain and works as a gateway keeper regulating and integrating sensory information. Although, it has been proposed that rolandic spikes could be generated within the thalamo-cortical circuitry [25-27], most studies conducted in RE patients with no cognitive deficits did not report findings in cortical-subcortical network [10,28] except in [29]. It is important to 
stress that these studies applied a different methodology than network analysis, in addition, there is a lack of network studies in RE.

The network analysis was complemented by an exploratory HRF study. We found differences in the HRF height confirming an inhomogeneity in the hemodynamic response in the thalamus among groups. The height of HRF on left thalamus was up to 3 times bigger in the IED group when compared to the nonIEDs. This difference in height shown here reflects the neural and vascular changes resulting from spontaneous neural activity. Analyses with canonical HRF showed no differences between groups. It is an additional evidence that canonical HRF may not be the best model for RE, especially for subcortical regions as also shown by previous studies [10-14].

Altogether, we provide another experimental evidence for the involvement of thalamus and generation of spikes in RE, supporting the hypothesis that dysfunction in thalamo-cortical part of the somatosensory system might play a role in the pathophysiology of RE [25-27]. It has been shown that intrinsic activity in the thalamus is correlated to the number of IEDs the recent study [29]. In epilepsy, seizures are often associated with thalamo-cortical system [25,30-33]. It has been suggested that the thalamus would play an important role in source synchronization and oscillatory activity in non-REM sleep [26]. Interestingly, in RE seizures usually occur at night during sleep.

In addition, our results showed hyper excitability away from the expected focal cortical seed located in rolandic area. This represents an additional evidence that RE is not solely a focal localized disease and should rather be thought as disease that affects complex networks in the brain [34]. The role of the thalamus could be further confirmed in meta analysis as the small group size is the main limitation of this study. To the best of our knowledge, this is the first study to combine blind deconvolution and voxel wise network analysis by FCD to the whole brain of RE patients.

\section{Acknowledgments}

The work has been supported by Conselho Nacional de Desenvolvimento Científico e Tecnológico CNPq.

\section{Compliance with Ethical Standards}

Conflict of Interest: The authors declare that they have no conflict of interest.

For this type of study formal consent is not required.

\section{References}

1. Panayiotopoulos CP, Michael M, Sanders S, Valeta T, Koutroumanidis M (2008) Benign childhood focal epilepsies: Assessment of established and newly recognized syndromes. Brain 131: 2264-2286. doi:10.1093/brain/awn162.

2. Kavros PM, Clarke T, Strug LJ, Halperin JM, Dorta NJ, et al. (2008) Attention impairment in rolandic epilepsy: systematic review. Epilepsia 49: 1570-1580.

3. Overvliet GM, Besseling RMH, Vles JSH, Hofman P a M, Backes WH, et al. (2010) Nocturnal epileptiform EEG discharges, nocturnal epileptic seizures, and language impairments in children: Review of the literature. Epilepsy Behav 19: 550-558.

4. Doesburg SM, Ibrahim GM, Smith M Lou, Sharma R, Viljoen A, et al. (2013) Altered Rolandic gamma-band activation associated with motor impairment and ictal network desynchronization in childhood epilepsy. PLoS One 8: e54943.

5. Verrotti A, Filippini M, Matricardi S, Agostinelli MF, Gobbi G (2014) Memory impairment and Benign Epilepsy with 
centrotemporal spike (BECTS): A growing suspicion. Brain Cogn 84: 123-131.

6. Besseling RMH, Overvliet GM, Jansen JF a, van der Kruijs SJM, Vles JSH, et al. (2013) Aberrant functional connectivity between motor and language networks in rolandic epilepsy. Epilepsy Res 107: 253-262.

7. Massa R, de Saint-Martin A, Carcangiu R, Rudolf G, Seegmuller C, et al. (2001) EEG criteria predictive of complicated evolution in idiopathic rolandic epilepsy. Neurology 57: 1071-1079.

8. Glover GH (1999) Deconvolution of impulse response in event-related BOLD fMRI. Neuroimage 9: 416-429.

9. Ashby FG (2011) Statistical analysis of fMRI data. MIT Press. 332 p.

10. Masterton RAJ, Harvey AS, Archer JS, Lillywhite LM, Abbott DF, et al. (2010) Focal epileptiform spikes do not show a canonical BOLD response in patients with benign rolandic epilepsy (BECTS). Neuroimage 51:252-260.

11. Lu Y, Bagshaw AP, Grova C, Kobayashi E, Dubeau F, et al. (2006) Using voxel-specific hemodynamic response function in EEG-fMRI data analysis. Neuroimage 32: 238-247. doi:10.1016/j.neuroimage.2005.11.040.

12. Jacobs J, Hawco C, Kobayashi E, Boor R, LeVan P, et al. (2008) Variability of the hemodynamic response as a function of age and frequency of epileptic discharge in children with epilepsy. Neuroimage 40: 601-614.

13. Lemieux L, Laufs H, Carmichael D, Paul JS, Walker MC, et al. (2007) Noncanonical spike-related BOLD responses in focal epilepsy. Hum Brain Mapp 29: 329-345.

14. Pellegrino G, Machado A, von Ellenrieder N, Watanabe S, Hall JA, et al. (2016) Hemodynamic Response to Interictal Epileptiform Discharges Addressed by Personalized EEG-fNIRS Recordings. Front Neurosci 10.

15. Wu G-RG, Liao W, Stramaglia S, Ding J-RJ, Chen H, et al. (2013) A blind deconvolution approach to recover effective connectivity brain networks from resting state fMRI data. Med image 17: 365-374.

16. Wu G-R, Marinazzo D (2015) Point-Process Deconvolution of fMRI BOLD Signal Reveals Effective Connectivity Alterations in Chronic Pain Patients. Brain Topogr 28: 541-547.

17. Tomasi D, Wang R, Wang G-J, Volkow ND (2014) Functional connectivity and brain activation: a synergistic approach. Cereb Cortex 24: 2619-2629.

18. Tomasi D, Volkow ND (2010) Functional connectivity density mapping. Proc Natl Acad Sci U S A 107: 9885-9890. doi:10.1073/pnas.1001414107.

19. Berg AT, Berkovic SF, Brodie MJ, Buchhalter J, Cross JH, et al. (2010) Revised terminology and concepts for organization of seizures and epilepsies: Report of the ILAE Commission on Classification and Terminology, 2005-2009. Epilepsia 51: 676-685. doi:10.1111/j.1528-1167.2010.02522.x.

20. Yan C, Zang Y (2010) DPARSF: a MATLAB toolbox for "pipeline" data analysis of resting-state fMRI. Front Syst Neurosci 4: 13 .

21. Song X-W, Dong Z-Y, Long X-Y, Li S-F, Zuo X-N, et al. (2011) REST: a toolkit for resting-state functional magnetic resonance imaging data processing. PLoS One 6: e25031.

22. Power JD, Barnes KA, Snyder AZ, Schlaggar BL, Petersen SE (2012) Spurious but systematic correlations in functional connectivity MRI networks arise from subject motion. Neuroimage 59: 2142-2154.

23. Deco G, Jirsa VK (2012) Ongoing cortical activity at rest: criticality, multistability, and ghost attractors. J Neurosci 32 : 3366-3375.

24. Tagliazucchi E, Balenzuela P, Fraiman D, Montoya P, Chialvo DR (2011) Spontaneous BOLD event triggered averages for estimating functional connectivity at resting state. Neurosci Lett 488: 158-163.

25. Avanzini G, Manganotti P, Meletti S, Moshé SL, Panzica F, et al. (2012) The system epilepsies: a pathophysiological hypothesis. Epilepsia 53: 771-778.

26. Kellaway P (2000) The Electroencephalographic Features of Benign Centrotemporal (Rolandic) Epilepsy of Childhood. Epilepsia 41: 1053-1056.

27. Huguenard JR (2000) Circuit mechanisms of spike-wave discharge: are there similar underpinnings for centrotemporal spikes? Epilepsia 41: 1076-1077.

28. Boor R, Jacobs J, Hinzmann A, Bauermann T, Scherg M, et al. (2007) Combined spike-related functional MRI and multiple source analysis in the non-invasive spike localization of benign rolandic epilepsy. Clin Neurophysiol 118: 901-909.

29. Zhu Y, Yu Y, Shinkareva S V, Ji G-J, Wang J, et al. (2015) Intrinsic brain activity as a diagnostic biomarker in children with benign epilepsy with centrotemporal spikes. Hum Brain Mapp 36: 3878-3889.

30. Carney PW, Jackson GD (2014) Insights into the Mechanisms of Absence Seizure Generation Provided by EEG with Functional MRI. Front Neurol 5: 1-13.

31. Marshall WJ, Lackner CL, Marriott P, Santesso DL, Segalowitz SJ (2014) Using phase shift Granger causality to measure directed connectivity in EEG recordings. Brain Connect 4: 826-841.

32. Vaudano AE, Laufs H, Kiebel SJ, Carmichael DW, Hamandi K, et al. (2009) Causal Hierarchy within the Thalamo-Cortical Network in Spike and Wave Discharges. PLoS One 4: e6475.

33. Lemieux L, Daunizeau J, Walker MC (2011) Concepts of connectivity and human epileptic activity. Front Syst Neurosci 5: 12.

34. Centeno M, Carmichael DW (2014) Network connectivity in epilepsy: Resting state fMRI and EEG-fMRI contributions. Front Neurol 5 JUL. doi:10.3389/fneur.2014.00093. 